July 2014

Atkinson and Stiglitz theorem in the presence of a household production sector

Helmuth Cremer and Firouz Gahvari 


\title{
Atkinson and Stiglitz theorem in the presence of a household production sector
}

\author{
Helmuth Cremer \\ Toulouse School of Economics \\ (University of Toulouse and Institut universitaire de France) \\ Toulouse, France \\ Firouz Gahvari \\ Department of Economics \\ University of Illinois at Urbana-Champaign \\ Urbana, IL 61801, USA
}

July 2014 


\begin{abstract}
We show that the celebrated Atkinson and Stiglitz (1976) result on the uniformity of the commodity tax rates when preferences are weakly separable between goods and leisure does not hold when (at least) one of the goods is produced within the household. The result is restored if preferences are weakly separable in market goods on the one hand, and leisure and household goods on the other.
\end{abstract}

JEL classification: $\mathrm{H} 2, \mathrm{H} 5$.

Keywords: Atkinson and Stiglitz theorem, household production, weak-separability. 


\section{Introduction}

In their classic contribution, Atkinson and Stiglitz (1976) prove that if preferences are weakly separable in labor supply and goods, an optimal nonlinear income tax is sufficient to implement any incentive compatible Pareto-efficient allocation. Put differently, commodity taxes are redundant or should be uniform. The Atkinson and Stiglitz (AS) result has had a tremendous effect in shaping the views of public economists concerning the design of optimal tax systems. This includes the widely-held belief that prices should not be used for redistribution (even in a second best setting), and that in-kind transfers are not useful. ${ }^{1}$

First. we show that when (at least) one of the goods is produced within the household (referred to as a "household good"), the weak-separability in labor supply and goods is no longer sufficient to yield uniform commodity taxes. This is because the various marginal rates of substitution between goods are no longer independent of labor/leisure. Separability in leisure notwithstanding, the marginal rates of substitution depend on the quantity of the household good consumed. This opens up a second channel through which leisure affects these marginal rates of substitution. Secondly, we prove that the AS theorem can be restored under a slightly different separability condition. This is when preferences are weakly-separable in market goods on the one hand, and leisure and household goods on the other.

To establish these results, we consider the realistic mixed taxation setting à la Christiansen (1984) that combines nonlinear income taxation with linear pricing (taxation) of consumption goods. The results will hold a fortiori if goods can also be taxed nonlinearly.

\footnotetext{
${ }^{1}$ Another implication is that the Ramsey tax results, which typically imply non-uniform commodity taxes, come about merely as an artifact of restricting the income tax to be linear; an ad hoc and inconsistent assumption given the assumed information structure.
} 


\section{The model}

There are $H$ types of individuals, indexed $j=1, \ldots, H$, who differ in their wages, $w^{j}$, but have identical preferences over goods and leisure. One of the goods, $x_{0}$, is produced within the household, using internal labor, and not sold on the market. Hence it is also non-taxable. All market goods, $\underline{x}=\left(x_{1}, x_{2}, \ldots, x_{n}\right)$, are produced at a constant marginal cost which we normalize to one. Let $\underline{p}=\left(p_{1}, p_{2}, \ldots, p_{n}\right)$ denote the consumer price of $\underline{x}$. We have $p_{i}=1+t_{i}(i=1,2, \ldots, n)$, where $\underline{t}=\left(t_{1}, t_{2}, \ldots, t_{n}\right)$ denote the commodity taxes.

Individual consumption levels are not publicly observable but anonymous transactions can be observed. Hence commodity taxes must be proportional and public sector prices are linear. For the remaining variables, the information structure is the one typically considered in mixed taxation models; see e.g., Christiansen (1984) and Cremer and Gahvari (1997). In particular, an individual's type, $w^{j}$, and labor input, $L^{j}$, are not publicly observable; his before-tax income, $I^{j}=w^{j} L^{j}$, on the other hand, is. Consequently, type-specific lump-sum taxation is ruled out but non-linear taxation of incomes is feasible.

To characterize the (constrained) Pareto-efficient allocations we derive an optimal revelation mechanism. For our purpose, a mechanism consists of a set of type-specific before-tax incomes, $I^{j}$ 's, aggregate expenditures on private sector goods, $c^{j}$ 's, and the vector of consumer prices (same for everyone) $\underline{p}$.

To proceed further, it is necessary to consider the optimization problem of an individual for a given mechanism $(p, c, I)$. With good 0 produced in the household, this requires some care. Denote leisure time by $l$ and an individual's time endowment by $T$. When a $j$-type individual spends $l_{0}$ of his time to produce $x_{0}$ units of the household good, he will be left with $l=T-L-l_{0}$ units of leisure. One can then represent a $j$-type individual's preferences over $x_{0}, \underline{x}$ and $l$ by means of the utility function $U=U\left(x_{0}, \underline{x}, T-L-l_{0}\right)$. To be realistic, assume that $x_{0}=\alpha w^{j} l_{0}$ so that $x_{0}$ varies not only with $l_{0}$ but also 
with the individual's productivity $w^{j} .^{2}$ We can then represent the $j$-type individual's preferences by

$$
\begin{aligned}
U\left(x_{0}, \underline{x}, T-L-l_{0}\right) & =U\left(x_{0}, \underline{x}, T-I / w^{j}-x_{0} / \alpha w^{j}\right) \\
& \equiv u\left(x_{0}, \underline{x}, I ; w^{j}\right) .
\end{aligned}
$$

Formally, given any vector $(\underline{p}, c, I)$, an individual of type $j$ chooses $x_{0}$ and $\underline{x}$ to maximize his utility $u=u\left(x_{0}, \underline{x}, I ; w^{j}\right)$ subject to the budget constraint $\sum_{i=1}^{n} p_{i} x_{i}=c$. The first-order conditions between market goods are standard yielding

$$
\frac{\partial u / \partial x_{i}}{\partial u / \partial x_{1}}=\frac{\partial U / \partial x_{i}}{\partial U / \partial x_{1}}=\frac{p_{i}}{p_{1}}, \quad i=2,3, \ldots, n .
$$

On the other hand, the first-order condition for $x_{0}$ is given by

$$
\frac{\partial u}{\partial x_{0}}=\frac{\partial U}{\partial x_{0}}-\frac{1}{\alpha w^{j}} \frac{\partial U}{\partial l}=0 .
$$

Solving equations (1)-(2), along with the budget constraint $\sum_{i=1}^{n} p_{i} x_{i}=c$, leads to the demand functions for $x_{0}$ and $\underline{x}$; they are denoted by $x_{0}=x_{0}\left(\underline{p}, c, I ; w^{j}\right)$ and $x_{i}=x_{i}\left(\underline{p}, c, I ; w^{j}\right)$. Substituting them in the utility function $u\left(x_{0}, \underline{x}, I ; w^{j}\right)$ yields the indirect utility function $v\left(\underline{p}, c, I ; w^{j}\right) \equiv u\left[x_{0}\left(\underline{p}, c, I ; w^{j}\right), \underline{x}\left(\underline{p}, c, I ; w^{j}\right), I ; w^{j}\right]$. Thus, a $j$-type individual who is assigned $c^{j}, I^{j}$ will have demand functions and an indirect utility function given by

$$
x_{0}^{j}=x_{0}\left(\underline{p}, c, I ; w^{j}\right), x_{i}^{j}=x_{i}\left(\underline{p}, c^{j}, I^{j} ; w^{j}\right), \quad \text { and } \quad v^{j}=v\left(\underline{p}, c^{j}, I^{j} ; w^{j}\right) .
$$

Similarly, the demand functions and the indirect utility function for a $j$-type who claims to be of type $k$, the so-called mimicker, is given by

$$
x_{0}^{j k}=x_{0}\left(\underline{p}, c^{k}, I^{k} ; w^{j}\right), x_{i}^{j k}=x_{i}\left(\underline{p}, c^{k}, I^{k} ; w^{j}\right), \quad \text { and } \quad v^{j k}=v\left(\underline{p}, c^{k}, I^{k} ; w^{j}\right) .
$$

\footnotetext{
${ }^{2}$ It goes without saying that this assumption has no bearing on our results.
} 


\subsection{Pareto-efficient (constrained) allocations}

Denote the government's external revenue requirement by $\bar{R}$. Constrained Paretoefficient allocations are described, indirectly, as follows. ${ }^{3}$ Maximize

$$
\sum_{j=1}^{H} \eta^{j} v\left(\underline{p}, c^{j}, I^{j} ; w^{j}\right)
$$

with respect to $\underline{p}, c^{j}$ and $I^{j}$, where $\eta^{j}$ s are constants with the normalization $\sum_{j=1}^{H} \eta^{j}=$ $1 .^{4}$ The maximization is subject to the resource constraint

$$
\sum_{j=1}^{H} \pi^{j}\left[\left(I^{j}-c^{j}\right)+\sum_{i=1}^{n}\left(p_{i}-1\right) x_{i}^{j}\right] \geq \bar{R}
$$

and the self-selection constraints

$$
v^{j} \geq v^{j k}, \quad j, k=1,2, \ldots, H
$$

Denote the Lagrangian expression by $\mathcal{L}$, and the Lagrangian multipliers associated with the resource constraint (6) by $\mu$, and with the self-selection constraints (7) by $\lambda^{j k}$. We have

$$
\mathcal{L}=\sum_{j} \eta^{j} v^{j}+\mu\left\{\sum_{j} \pi^{j}\left[\left(I^{j}-c^{j}\right)+\sum_{i=1}^{n}\left(p_{i}-1\right) x_{i}^{j}\right]-\bar{R}\right\}+\sum_{j} \sum_{k \neq j} \lambda^{j k}\left(v^{j}-v^{j k}\right) .
$$

The first-order conditions of this problem with respect to $I^{j}, c^{j}$, for $j, k=1,2, \ldots, H$, and $p_{i}$, for $i=1,2, \ldots, n$, characterize the Pareto-efficient allocations constrained by the resource constraint, the self-selection constraints, and the linearity of commodity taxes.

\footnotetext{
${ }^{3}$ Indirectly because the optimization is over a mix of quantities and prices. Upon the determination of the commodity prices, utility-maximizing individuals would choose the quantities themselves.

${ }^{4}$ Recall that $x_{0}$ is non-taxable so that one cannot optimize over its consumer price $p_{0}$.
} 


\section{Atkinson and Stiglitz theorem and optimal commodity taxes}

Denote, $\forall i, j, k$, the compensated demand corresponding to $x_{i}^{j}$ by $\tilde{x}_{i}^{j}$ and introduce

$$
\Delta \equiv\left(\begin{array}{cccc}
\sum_{j} \pi^{j} \frac{\partial \tilde{x}_{1}^{j}}{\partial p_{1}} & \sum_{j} \pi^{j} \frac{\partial \tilde{x}_{1}^{j}}{\partial p_{2}} & \cdots & \sum_{j} \pi^{j} \frac{\partial \tilde{x}_{1}^{j}}{\partial p_{n}} \\
\sum_{j} \pi^{j} \frac{\partial \tilde{x}_{2}^{j}}{\partial p_{1}} & \sum_{j} \pi^{j} \frac{\partial \tilde{x}_{2}^{j}}{\partial p_{2}} & \cdots & \sum_{j} \pi^{j} \frac{\partial \tilde{x}_{2}^{j}}{\partial p_{n}} \\
\vdots & \vdots & \ddots & \vdots \\
\sum_{j} \pi^{j} \frac{\partial \tilde{x}_{n}^{j}}{\partial p_{1}} & \sum_{j} \pi^{j} \frac{\partial \tilde{x}_{n}^{j}}{\partial p_{2}} & \cdots & \sum_{j} \pi^{j} \frac{\partial \tilde{x}_{n}^{j}}{\partial p_{n}}
\end{array}\right) .
$$

Thus $\Delta$ denotes the $n \times n$ matrix derived from the $(n+1) \times(n+1)$ Slutsky matrix, aggregated over all individuals, by deleting its first row and column corresponding to the household good $x_{0}$. We prove in the Appendix that the optimal commodity taxes are given $b^{5}$

$$
\left(\begin{array}{c}
t_{1} \\
\vdots \\
t_{n}
\end{array}\right)=\frac{1}{\mu} \Delta^{-1}\left(\begin{array}{c}
\sum_{j} \sum_{k \neq j} \lambda^{k j}\left(x_{1}^{j}-x_{1}^{k j}\right) v_{c}^{k j} \\
\vdots \\
\sum_{j} \sum_{k \neq j} \lambda^{k j}\left(x_{n}^{j}-x_{n}^{k j}\right) v_{c}^{k j}
\end{array}\right)
$$

Expressions (10) show that whenever $x_{i}^{j}=x_{i}^{k j}, \forall i, j, k$, then all commodity taxes are equal to zero. ${ }^{6}$ That is, for tax purposes, market goods should be treated the same way as the good produced within the household.

\subsection{Absent household goods}

To set the stage for studying the structure of taxes in our setting, we begin by taking a detour to the traditional framework when there is no household good and all goods are produced in the market. To represent this case within our formulation, one needs only set $l_{0}=0$ while assuming that $x_{0}$ is a market good too. At first blush, however, this assumption appears to open up the possibility of levying a tax on good 0. Yet, as is well known in the optimal tax literature, under this circumstance, the optimal tax rates are

\footnotetext{
${ }^{5}$ Observe that $\Delta$ is of full rank so that its inverse exists; see Takayama (1985).

${ }^{6}$ This is sufficient but not necessary; a weaker condition requires that $x_{i}^{j}=x_{i}^{k j}$ whenever $\lambda^{k j}>0$.
} 
not unique. The reason is that with all goods being purchased in the market, $x_{0}$ and $\underline{x}$ become homogeneous of degree zero in market prices and income: $p_{0}, \underline{p}$, and $c$. This means that consumer prices can be determined only up to a proportionality factor.

To deal with this issue, the literature typically normalizes one of the consumer prices to one. Following this procedure, one may just as well choose good 0 to be the good whose price is fixed and normalized to one. With $p_{0}=1$, tax optimization is carried out, as in our setting, over $i=1,2, \ldots, n$ but not $i=0$. Consequently, one retrieves our previous result for the optimal tax rates given by (10). Moreover, in this case, weak-separability, $U=U\left(f\left(x_{0}, \underline{x}\right), l\right)$, in conjunction with optimization over $x_{0}$ and $\underline{x}$ subject to the budget constraint $\sum_{i=0}^{n} p_{i} x_{i}=c$, implies

$$
\frac{\partial u / \partial x_{i}}{\partial u / \partial x_{0}}=\frac{\partial U / \partial x_{i}}{\partial U / \partial x_{0}}=\frac{\partial f / \partial x_{i}}{\partial f / \partial x_{0}}\left(x_{0}, \underline{x}\right)=\frac{p_{i}}{p_{0}}, \quad i=1,2, \ldots, n .
$$

It follows from solving the above equations along with the budget constraint $\sum_{i=0}^{n} p_{i} x_{i}=$ $c$ that the demand functions for all goods including $x_{0}$ are independent of leisure. Consequently, with individuals $j$ and $k$ mimicking $j$, having the same disposable income $c^{j}$ (and facing the same prices), their demands will be identical. This delivers the AS theorem for the traditional setting with no household production.

\subsection{The presence of household goods}

We now return to our original setting with $x_{0}$ being produced within the household. It is rather obvious that in this case, weak-separability of preferences between goods and leisure no longer ensures that commodity taxes are zero. With $U=U\left(f\left(x_{0}, \underline{x}\right), l\right)$, equations (1)-(2) are simplified to

$$
\begin{aligned}
& \frac{\partial U / \partial x_{i}}{\partial U / \partial x_{1}}=\frac{\partial f / \partial x_{i}}{\partial f / \partial x_{1}}\left(x_{0}, \underline{x}\right)=\frac{p_{i}}{p_{1}}, i=2,3, \ldots, n, \\
& \frac{\partial U}{\partial x_{0}}-\frac{1}{\alpha w^{j}} \frac{\partial U}{\partial l}=\frac{\partial U}{\partial f} \frac{\partial f}{\partial x_{0}}-\frac{1}{\alpha w^{j}} \frac{\partial U}{\partial l}=0 .
\end{aligned}
$$

If one now were to "solve" equations (11) along with the budget constraint $\sum_{i=1}^{n} p_{i} x_{i}=c$ for $\underline{x}$, the resulting equations will be dependent on $x_{0}$. But simple inspection of (12) 
reveals that $x_{0}$ depends on $l$. Hence the demand functions for $\underline{x}$ will also depend on $l$. As a consequence, the $j$-type individual's demand for good $i$ will depend on his wage $w^{j}$ (whether he reports his type to be $j$ or $k \neq j$ ). Thus, whereas the $j$-type individual's demand for good $i$ depends on $w^{j}$, the "mimicker's" demand - the demand by a $k$-type who reports his type as $j$-will be a function of $w^{k}$. It then follows that in general $x_{i}^{j} \neq x_{i}^{k j}$ so that, from (10), the Atkinson and Stiglitz result breaks down. In words, when one of the goods is produced in the household, weak-separability of preferences between goods and leisure is no longer sufficient to make commodity taxation redundant.

Next, we show that the AS theorem can be restored under a slightly different separability condition. Assume that preferences are weakly-separable in market goods on the one hand, and leisure and household goods on the other. One can then rewrite the utility function $u\left(x_{0}, \underline{x}, I ; w^{j}\right)$ as $U\left(f(\underline{x}), g\left(x_{0}, I ; w^{j}\right)\right) .{ }^{7}$ Under this circumstance, the (conditional) demand functions for $\underline{x}$, as specified in $(3)$, will be independent of $\left(I ; w^{j}\right)$ so that $x_{i}=x_{i}(\underline{p}, c)$. To see this, observe that given $I$, maximization of the direct utility function subject to the budget constraint $\sum_{i=1}^{n} p_{i} x_{i}=c$ now yields

$$
\frac{\partial U / \partial x_{i}}{\partial U / \partial x_{1}}=\frac{\partial f / \partial x_{i}}{\partial f / \partial x_{1}}(\underline{x})=\frac{p_{i}}{p_{1}}, \quad i=2,3, \ldots, n .
$$

With (13) being independent of $x_{0}$, "solving" these $(n-1)$ equations, alongside the budget constraint $\sum_{i=1}^{n} p_{i} x_{i}=c$, results in demand functions for $\underline{x}$ which depend only on $(\underline{p}, c)$ and not on $\left(I ; w^{j}\right)$. Consequently equations (10) imply, once again, that all commodity taxes are equal to zero. Of course, the demand for household good $x_{0}$ will

\footnotetext{
${ }^{7}$ This follows because, with separability, the direct utility function is written as

$$
\begin{aligned}
U\left(x_{0}, \underline{x}, l\right) & =U\left(f(\underline{x}), h\left(x_{0}, l\right)\right)=U\left(f(\underline{x}), h\left(x_{0}, T-L-l_{0}\right)\right) \\
& =U\left(f(\underline{x}), h\left(x_{0}, T-I / w^{j}-x_{0} / \alpha w^{j}\right)\right) \\
& =U\left(f(\underline{x}), g\left(x_{0}, I ; w^{j}\right)\right) \\
& \equiv u\left(x_{0}, \underline{x}, I ; w^{j}\right) .
\end{aligned}
$$
}


depend on a person's type and his assigned income. ${ }^{8}$ However, under the considered type of separability, this has no impact on the conditional demands for market goods $\underline{x}$.

\section{Conclusion}

This note has shown that the celebrated AS result, stating that commodity taxes should be uniform if preferences are weakly separable between goods and leisure, does not apply when (at least) one of the goods is produced within the household. On the other hand, the AS theorem can be restored if preferences are weakly separable in market goods on the one hand, and leisure and household goods on the other.

Finally, we should point out in conclusion that our results hold regardless of how many household goods there are. While there was only one household good in our formulation, this was simply for expositional ease. A quick inspection of equations (13) shows clearly that the separability condition considered is sufficient for uniform commodity taxes even if $x_{0}$ is a vector comprising two or more household goods. Of course, this requires that all household goods to be weakly separable from the market goods.

${ }^{8}$ The first-order condition in maximization of $U(\cdot)$ with respect to $x_{0}$ is

$$
\frac{\partial U}{\partial x_{0}}=\frac{\partial U}{\partial g} \frac{\partial g}{\partial x_{0}}=\frac{\partial U}{\partial g}\left[\frac{\partial h}{\partial x_{0}}-\frac{1}{\alpha w^{j}} \frac{\partial h}{\partial l}\right]=0 .
$$

where $h=h\left(x_{0}, T-I / w^{j}-x_{0} / \alpha w^{j}\right)$. Consequently,

$$
\frac{\partial h}{\partial x_{0}}\left(x_{0}, T-I / w^{j}-x_{0} / \alpha w^{j}\right)=\frac{1}{\alpha w^{j}} \frac{\partial h}{\partial l}\left(x_{0}, T-I / w^{j}-x_{0} / \alpha w^{j}\right) .
$$

Solving this equation for $x_{0}$ yields

$$
x_{0}=x_{0}\left(I ; w^{j}\right) .
$$

Observe also that this implies

$$
x_{0}^{k}=x_{0}\left(I^{k} ; w^{k}\right) \text { and } x_{0}^{j k}=x_{0}\left(I^{k} ; w^{j}\right) .
$$

Hence

$$
x_{0}^{j k} \neq x_{0}^{k}
$$




\section{Appendix}

First-order characterization of the(constrained) Pareto-efficient allocations: Rearranging the terms in (8), and dropping the constant, one may usefully rewrite the Lagrangian expression as

$$
\mathcal{L}=\sum_{j}\left(\eta^{j}+\sum_{k \neq j} \lambda^{j k}\right) v^{j}+\mu \sum_{j} \pi^{j}\left[\left(I^{j}-c^{j}\right)+\sum_{i=1}^{n}\left(p_{i}-1\right) x_{i}^{j}\right]-\sum_{j} \sum_{k \neq j} \lambda^{j k} v^{j k} .
$$

The first-order conditions of this problem are, for $j, k=1,2, \ldots, H$,

$$
\begin{aligned}
\frac{\partial \mathcal{L}}{\partial I^{j}}= & \left(\eta^{j}+\sum_{k \neq j} \lambda^{j k}\right) v_{I}^{j}+\mu \pi^{j}\left[1+\sum_{i=1}^{n}\left(p_{i}-1\right) \frac{\partial x_{i}^{j}}{\partial I^{j}}\right]-\sum_{k \neq j} \lambda^{k j} v_{I}^{k j}=0, \\
\frac{\partial \mathcal{L}}{\partial c^{j}}= & \left(\eta^{j}+\sum_{k \neq j} \lambda^{j k}\right) v_{c}^{j}+\mu \pi^{j}\left[-1+\sum_{i=1}^{n}\left(p_{i}-1\right) \frac{\partial x_{i}^{j}}{\partial c^{j}}\right]-\sum_{k \neq j} \lambda^{k j} v_{c}^{k j}=0, \\
\frac{\partial \mathcal{L}}{\partial p_{i}}= & \sum_{j}\left(\eta^{j}+\sum_{k \neq j} \lambda^{j k}\right) v_{i}^{j}+\mu \sum_{j} \pi^{j}\left[\sum_{e=1}^{n}\left(p_{e}-1\right) \frac{\partial x_{e}^{j}}{\partial p_{i}}+x_{i}^{j}\right] \\
& -\sum_{j} \sum_{k \neq j} \lambda^{j k} v_{i}^{j k}=0, \quad i=1,2, \ldots, n,
\end{aligned}
$$

where a subscript on $v^{j}$ denotes a partial derivative. Equations (A2)-(A4) characterize the Pareto-efficient allocations constrained by the information structure.

Optimal commodity taxes: Multiply equation (A3) by $x_{i}^{j}$, sum over $j$ and add the resulting equation to (A4). Simplifying results in the following system of equations for $i=1,2, \ldots, n$,

$$
\begin{aligned}
& \frac{\partial \mathcal{L}}{\partial p_{i}}+\sum_{j} x_{i}^{j} \frac{\partial \mathcal{L}}{\partial c^{j}}=\sum_{j}\left(\eta^{j}+\sum_{k \neq j} \lambda^{j k}\right)\left(v_{i}^{j}+x_{i}^{j} v_{c}^{j}\right)+ \\
& \mu \sum_{j} \pi^{j}\left[\sum_{e=1}^{n}\left(p_{e}-1\right)\left(\frac{\partial x_{e}^{j}}{\partial p_{i}}+x_{i}^{j} \frac{\partial x_{e}^{j}}{\partial c^{j}}\right)\right]-\sum_{j} \sum_{k \neq j} \lambda^{k j}\left(v_{i}^{k j}+x_{i}^{j} v_{c}^{k j}\right)=0 .
\end{aligned}
$$

The left-hand side of (A5) shows the impact on the Lagrangian expression $\mathcal{L}$ of a variation in $p_{i}$ when the disposable income of individuals is adjusted according to $d c_{j}=x_{i}^{j} d t_{i}$, 
to keep their utility levels constant. These compensated derivatives, $\left(\partial \mathcal{L} / \partial p_{i}\right)_{v^{j}=\bar{v}^{j}}$, vanish at the optimal solution.

Make use of Roy's identity to set $v_{i}^{j}+x_{i}^{j} v_{c}^{j}=0$ and $v_{i}^{k j}+x_{i}^{k j} v_{c}^{k j}=0$ in (A5), replace $p_{i}-1$ by $t_{i}$, and divide by $\mu$. Upon changing the order of summation and further simplification one arrives at, for all $i=1,2, \ldots, n$,

$$
\sum_{e=1}^{n} t_{e}\left[\sum_{j} \pi^{j}\left(\frac{\partial x_{e}^{j}}{\partial p_{i}}+x_{i}^{j} \frac{\partial x_{e}^{j}}{\partial c^{j}}\right)\right]-\frac{1}{\mu} \sum_{j} \sum_{k \neq j} \lambda^{k j}\left(x_{i}^{j}-x_{i}^{k j}\right) v_{c}^{k j}=0 .
$$

Using the Slutsky equation,

$$
\frac{\partial x_{e}^{j}}{\partial p_{i}}=\frac{\partial \tilde{x}_{e}^{j}}{\partial p_{i}}-x_{i}^{j} \frac{\partial x_{e}^{j}}{\partial c^{j}},
$$

and making use of the symmetry of the Slutsky matrix, one can further simplify (A6) to

$$
\sum_{e=1}^{n} t_{e}\left(\sum_{j} \pi^{j} \frac{\partial \tilde{x}_{i}^{j}}{\partial p_{e}}\right)=\frac{1}{\mu} \sum_{j} \sum_{k \neq j} \lambda^{k j}\left(x_{i}^{j}-x_{i}^{k j}\right) v_{c}^{k j}
$$

which holds for all $i=1,2, \ldots, n$. Then using the definition of $\Delta$ in (9) one can rewrite equations (A7) in matrix notation as

$$
\Delta\left(\begin{array}{c}
t_{1} \\
\vdots \\
t_{n}
\end{array}\right)=\frac{1}{\mu}\left(\begin{array}{c}
\sum_{j} \sum_{k \neq j} \lambda^{k j}\left(x_{1}^{j}-x_{1}^{k j}\right) v_{c}^{k j} \\
\vdots \\
\sum_{j} \sum_{k \neq j} \lambda^{k j}\left(x_{n}^{j}-x_{n}^{k j}\right) v_{c}^{k j}
\end{array}\right)
$$

Premultiplying (A8) through by $\Delta^{-1}$ yields (10).

\section{References}

[1] Atkinson, Anthony B. and Joseph E. Stiglitz, 1976. The design of tax structure: direct versus indirect taxation, Journal of Public Economics, 6, 55-75.

[2] Christiansen, Vidar, 1984. Which commodity taxes should supplement the income tax? Journal of Public Economics, 24, 195-220. 